\title{
FOR $n>3$ THERE IS ONLY ONE \\ FINITELY ADDITIVE ROTATIONALLY INVARIANT MEASURE ON THE $n$-SPHERE DEFINED \\ ON ALL LEBESGUE MEASURABLE SUBSETS
}

BY DENNIS SULLIVAN

The following paragraph is taken from the introduction of Joseph Rosenblatt's paper $[\mathbf{R}]$.

"Let $\beta$ be the ring of Lebesgue measurable sets in the $n$-sphere $S^{n}$, and let $\lambda_{n}$ denote the Lebesgue measure on $\beta$ normalized by $\lambda_{n}\left(S^{n}\right)=1$. The classical characterization by Lebesgue of $\lambda_{n}$ is that it is the unique positive real-valued function $\mu$ on $\beta$ which satisfies these three conditions:

(a) $\mu\left(S^{n}\right)=1$;

(b) $\mu$ is invariant under isometries;

(c) $\mu$ is countably additive.

In 1923 Banach [B] studied the question of Ruziewicz whether $\mu$ is still unique when (c) is replaced by

$\left(c_{0}\right) \mu$ is finitely additive.

Banach gave a negative answer to this question for $S^{1}$ but for $S^{n}, n \geqslant 2$, the question is still unanswered."

From the body of Rosenblatt's paper one can extract the implication that if Lebesgue measure $\lambda_{n}$ on $S^{n}$ is not characterized by (a), (b), and $\left(\mathrm{c}_{0}\right)$ then there is a net of measurable subsets $\left(A_{\alpha}\right) \subset S^{2}$ which is asymptotically invariant and nontrivial, namely $\lim _{\alpha}\left(\lambda_{n}\left(g A_{\alpha} \Delta A_{\alpha}\right) / \lambda_{n} A_{\alpha}\right)=0$ for all rotations $g$ and so that $0<\lambda_{n}\left(A_{\alpha}\right) \leqslant c<1$ (Theorem 1.4 of $[\mathbf{R}]$ ). Here $A \Delta B=A \cup B-A \cap B$.

The following Proposition will show that such asymptotically invariant nets on $S^{n}$ are impossible, $n>3$.

Proposition. For each $n>3$ there is a countable subgroup $\Gamma_{n}$ in the group $O_{n+1}$ of rotations of $S^{n}$ satisfying

(i) the action of $\Gamma_{n}$ on $S^{n}$ is ergodic,

(ii) the group $\Gamma_{n}$ satisfies Kazhdan's property $T$ :

There exist a finite subset $\Lambda \subset \Gamma_{n}$ and an $\epsilon>0$, so that for any unitary representation $\pi$ if $\Gamma$, if there exists a vector $\zeta$ in $H_{\pi}$ such that $\|\zeta\|=1$,

Received by the editors July 28, 1980.

1980 Mathematics Subject Classification. Primary 28D10.

() 1981 American Mathematical Society $0002-9904 / 81 / 0000-0012 / \$ 01.75$ 
$\|\pi(g) \zeta-\zeta\| \leqslant \epsilon \forall g$ in $\Lambda$ then there exists a vector $\zeta^{\prime} \in H_{\pi}$ with $\pi(g) \zeta^{\prime}=\zeta^{\prime}$ $\forall g \in \Gamma_{n}$, and $\zeta^{\prime} \neq 0$.

Proof. For $n>3$ let $\Gamma_{n}$ be the group of $(n+1) \times(n+1)$ matrices with entries integers $(n+m \sqrt{2})$ of the field $Q(\sqrt{2})$ where such matrices preserve the quadratic form

$$
x_{0}^{2}+x_{1}^{2}+\cdots+x_{n-2}^{2}-\sqrt{2} x_{n-1}^{2}-\sqrt{2} x_{n}^{2} .
$$

If we conjugate all the matrices of $\Gamma_{n}$ by the field automorphism of $Q(\sqrt{2})$ we obtain a group of matrices isomorphic to $\Gamma_{n}$ preserving the form

$$
x_{0}^{2}+x_{1}^{2}+\cdots+x_{n-2}^{2}+\sqrt{2} x_{n-1}^{2}+\sqrt{2} x_{n}^{2} .
$$

So $\Gamma_{n}$ is embedded as a subgroup of $O(n+1)$, the real orthogonal group of the second quadratic form. If $O(n-1,2)$ denotes the real orthogonal group of the first quadratic form then the diagonal embedding $\Gamma_{n} \rightarrow O(n+1) \times$ $O(n-1,2)$ is discrete because the diagonal embedding $(n+m \sqrt{2}) \in Q(\sqrt{2}) \rightarrow$ $(n+m \sqrt{2}, n-m \sqrt{2}) \in R \times R$ is discrete. By a basic theorem of arithmetic groups $\Gamma_{n}$ has cofinite volume in $O(n+1) \times O(n-1,2)$. Since $O(n+1)$ is compact, $\Gamma_{n}$ is discrete with cofinite volume in $O(n-1,2)$.

Since $O(n-1,2)$ is a simple Lie group of real rank $\geqslant 2$ it has Kazhdan's property (see $[\mathbf{K}]$ ) which descends by an averaging argument (Theorem 3 of $[\mathbf{K}]$ ) to the discrete subgroup with cofinite volume $\Gamma_{n}$. Thus $\Gamma_{n}$ has Kazhdan's property $T$. This proves (ii).

Now if the topological closure of $\Gamma_{n} \subset O(n+1)$ were a proper closed subgroup $G$ then the complexification $G_{\mathrm{C}}$ of $G$ in the complexification $O(n+1, \mathbf{C})$ of $O(n+1)$ would define a proper $\mathrm{C}$-algebraic subgroup containing $\Gamma_{n}$. But for the conjugate embedding $\Gamma_{n} \subset O(n-1,2) \subset O(n+1, \mathbf{C}), \Gamma_{n}$ is Zariski dense by Borel's density theorem. This is a contradiction showing $\Gamma_{n}$ is topologically dense in $O(n+1)$.

Since $\Gamma_{n}$ is a dense subgroup of isometries ergodicity follows immediately from a consideration of Lebesgue density points. This proves (i).

Combining the Proposition with Rosenblatt's work [R] we have the answer to the Banach-Ruziewicz problem, $n>3$.

THEOREM. Spherical measure on $S^{n}, n>3$, is the only finitely additive normalized measure invariant under rotations and defined ${ }^{1}$ on all Lebesgue measurable sets.

Proof. If not by Rosenblatt [R] there is, as mentioned above, a nontrivial asymptotically invariant net of sets $\left(A_{\alpha}\right) \subset S^{2}$. Clearly we can extract a

\footnotetext{
${ }^{1}$ In $[R]$ one finds Tarski's observation using paradoxical decompositions that if a
} finitely additive measure is defined on all Lebesgue measurable sets it must be zero on Lebesgue null sets. 
countable subsequence $\left(A_{j}\right) \subset S^{2}$ which is asymptotically invariant for the countable subgroup $\Gamma_{n} \subset O_{n+1}$ constructed in the Proposition. Namely, $0<\lambda_{n}\left(A_{j}\right)$ $\leqslant c<1$, and for all $g \in \Gamma_{n} \lim _{j}\left(\lambda_{n}\left(g A_{j} \Delta A_{j}\right) / \lambda_{n}\left(A_{j}\right)\right)=0$.

Now convert the characteristic $f^{n s}$ of $A_{j}$ into functions of integral zero by forming $f_{j}=\left(\chi A_{j} / \sqrt{\lambda_{n}\left(A_{j}\right)}-\sqrt{\left.\lambda_{n}\left(A_{j}\right)\right)}\right.$ and then $F_{j}=f_{j} /\left\|f_{j}\right\|_{2}$. Then $\int F_{j} d \lambda_{n}=0$ because $\int f_{j} d \lambda_{n}=0$, and $\left\|F_{j}\right\|_{2}=1$. Also

$$
\left\|f_{j} \circ g-f_{j}\right\|_{2}^{2}=2\left(1-\lambda_{n}\left(g^{-1} A_{j} \cap A_{j}\right) / \lambda_{n}\left(A_{j}\right)\right) .
$$

Since $\left\|f_{\alpha}\right\|_{2}^{2}=\lambda_{n}\left(S^{2} \mid A_{j}\right)$, it is bounded away from zero by the nontriviality of $\left(A_{j}\right)$. Thus $\lim _{j}\left\|F_{j} \circ g-F_{j}\right\|_{2}=0$ for all $g \in \Gamma_{n}$ and $\left\|F_{j}\right\|_{2}=1$. (Compare [R, Lemma 3.1].)

If we apply property $T$ for $\Gamma_{n}$ for the representation of $\Gamma_{n}$ on the space $H$ of square integrable $f^{n s}$ on $S^{n}$ of integral zero we obtain from the existence of the vectors $F_{j}$ of $H$, the existence of an element in $H$ of norm 1 which is $\Gamma_{n}$ invariant. This contradicts ergodicity of $\Gamma_{n}$. Thus there is no such net of asymptotically invariant sets, and the Theorem is proved.

Acknowledgement. I am indebted to Arlan Ramsay for showing me Rosenblatt's paper during a conversation about the yet unsolved problem of the existence of a rotationally invariant finitely additive Borel measure on $S^{n}$ which is zero on meagre sets. (E. Marczewski (Szprilrajn), Problem 169, The Scottish Book 1937-1938). The realization that known examples of discrete groups having Kazhdan's property provided the answer to Ruziewicz's problem about Lebesgue measure occurred during a discussion of Rosenblatt's paper with Jan Mycielski.

\section{REFERENCES}

[B] S. Banach, Sur le problème de la mesure, S. Banach Oeuvres, vol I, Warszawa, 1967, pp. 318-322.

[K] D. A. Kazhdan, Connection of the dual space of a group with the structure of its closed subgroups, Functional Anal. Appl. 1 (1967), 63-65.

[R] J. Rosenblatt, Uniqueness of invariant means for measure preserving transformations, Trans. Amer. Math. Soc. (to appear).

DEPARTMENT OF MATHEMATICS, UNIVERSITY OF COLORADO, BOULDER, COLORADO 80309

Current address: Institut des Hautes Etudes Scientifiques, Bures-Yvette, France 